\title{
Three-Dimensional Dirac Oscillator with Minimal Length: Novel Phenomena for Quantized Energy
}

\author{
Malika Betrouche, ${ }^{1}$ Mustapha Maamache, ${ }^{2}$ and Jeong Ryeol Choi ${ }^{3}$ \\ ${ }^{1}$ Laboratoire de Physique Mathématique et Subatomique (lpmps), Départment de Physique, Faculté des Sciences Exactes, \\ Université Constantine1, 25000 Constantine, Algeria \\ ${ }^{2}$ Laboratoire de Physique Quantique et Systémes Dynamiques, Département de Physique, Faculté des Sciences, \\ Université Ferhat Abbas Sétif 1, 19000 Setif, Algeria \\ ${ }^{3}$ Department of Radiologic Technology, Daegu Health College, Yeongsong-ro 15, Daegu 702-722, Republic of Korea
}

Correspondence should be addressed to Jeong Ryeol Choi; choiardor@hanmail.net

Received 3 July 2013; Accepted 17 August 2013

Academic Editor: Gongnan Xie

Copyright (C) 2013 Malika Betrouche et al. This is an open access article distributed under the Creative Commons Attribution License, which permits unrestricted use, distribution, and reproduction in any medium, provided the original work is properly cited.

\begin{abstract}
We study quantum features of the Dirac oscillator under the condition that the position and the momentum operators obey generalized commutationrelations that lead to the appearance of minimal length with the order of the Planck length, $\Delta x_{\text {min }}=$ $\hbar \sqrt{3 \beta+\beta^{\prime}}$, where $\beta$ and $\beta^{\prime}$ are two positive small parameters. Wave functions of the system and the corresponding energy spectrum are derived rigorously. The presence of the minimal length accompanies a quadratic dependence of the energy spectrum on quantum number $n$, implying the property of hard confinement of the system. It is shown that the infinite degeneracy of energy levels appearing in the usual Dirac oscillator is vanished by the presence of the minimal length so long as $\beta \neq 0$. Not only in the nonrelativistic limit but also in the limit of the standard case $\left(\beta=\beta^{\prime}=0\right)$, our results reduce to well known usual ones.
\end{abstract}

\section{Introduction}

It is widely accepted that the consideration of a minimal length scale in nature, which is usually expected to be of the order of the Planck length, $l_{p}=\sqrt{G \hbar / c^{3}} \sim 10^{-35} \mathrm{~m}$, is necessary for a consistent formulation of quantum theory of gravity. According to this, the development of mathematical manipulation to handle the minimal length became a central issue in modern quantum gravity. The several research fields in which the concept of an observable minimal length plays an important role in their complete description are string theory [1-5], loop quantum gravity [6], noncommutative geometry [7], noncommutative field theories [8-10], and black-hole physics [11, 12]. Standard formulation of quantum mechanics with minimal length for these systems has been carried out starting from the modified Heisenberg algebra with a deformed commutation relation between position and momentum operators, which arises from intrinsic noncommutativity in geometries $[13,14]$. Other similar constructions that accompanies the concept of minimal length scale have also been initiated by some authors [15-18]. The introduction of minimal length scale leads to a generalization of the Heisenberg uncertainty principle in a way that it incorporates gravitationally induced uncertainty [19]. Kempf et al. introduced, through a series of their papers [20-23], a deformed quantum mechanics on the basis of modified commutation relations between position and momentum operators. These commutation relations are characterized with non-zero minimal length and lead to a generalized uncertainty principle (GUP) which includes some corrections from the ordinary Heisenberg uncertainty relation. There are plentiful reports for the consequences of the GUP. Among them, we quote the works of Kempf et al. [21, 22] and Chang et al. [24] that are relevant to the study for solving the Schrödinger equation in momentum space for the harmonic oscillator in $\mathrm{D}$-dimensions. Besides, the effects of the minimal length on the energy spectrum and momentum wave functions of the Coulomb potential in one dimension and three dimensions have been studied, respectively, in [25] and [26-28]. The quantization scheme in the presence of a minimal length 
is also applied to the problem of the Casimir force for the electromagnetic field $[29,30]$, the magnetization of electron [31], the Pauli equation for a charged particle in a magnetic field [32], and the cosmological constant problem [33, 34]. Further noteworthy studies in this direction include the high temperature properties of the one-dimensional Dirac oscillator (DO) [35] and supersymmetric quantum mechanics of the three-dimensional Dirac oscillator [14]. The Dirac oscillator plays an important role in the description of relativistic many-body problems and supersymmetric relativistic quantum mechanics [36-41]. Dirac oscillator representation is also proposed in quantum chromodynamics, particularly, in connection with quark confinement models in mesons and baryons [42].

The purpose of this work is to investigate the mathematical formulation of the Dirac oscillator problem and its consequences by solving fundamental equations in the framework of relativistic quantum mechanics with minimal length. In Section 2, we give a brief summary of the main features of quantum mechanics with generalized commutation relations. We solve, in Section 3, the three-dimensional Dirac oscillator equation in momentum space completely and derive the corresponding wave functions and the spectrum of quantized energy for $s=1 / 2$ (spin up) and $s=-1 / 2$ (spin down). The main consequences in the nonrelativistic limit are discussed and it is shown that our results reduce to the standard ones when we remove the scale of the minimal length. The concluding remarks are given in Section 4 which is the last section.

\section{Quantum Mechanics with Minimal Length}

The presence of a minimal length as a reflection of dynamical phenomenon stems from the fundamental fluctuations of the background metric at the Planck scales. The Planck length should be taken into account when we want to describe gravitational fields with its quantum fluctuations. Let us start this section with a brief review of deformed quantum mechanics in 3D. Indeed, following $[14,21]$, we have

$$
\begin{gathered}
{\left[\mathbf{x}_{i}, \mathbf{p}_{j}\right]=i \hbar\left[\delta_{i j}\left(1+\beta \mathbf{p}^{2}\right)+\beta^{\prime} \mathbf{p}_{i} \mathbf{p}_{j}\right],} \\
{\left[\mathbf{x}_{i}, \mathbf{x}_{j}\right]=-i \hbar\left(2 \beta-\beta^{\prime}+\left(2 \beta+\beta^{\prime}\right) \beta \mathbf{p}^{2}\right) \epsilon_{i j k} \mathbf{L}_{k},} \\
{\left[\mathbf{p}_{i}, \mathbf{p}_{j}\right]=0,}
\end{gathered}
$$

where $\beta$ and $\beta^{\prime}$ are two very small nonnegative parameters. The components of the angular momentum are given by

$$
\mathbf{L}_{i}=\left(1+\beta \mathbf{p}^{2}\right)^{-1} \epsilon_{i j k} \mathbf{x}_{j} \mathbf{p}_{k}, \quad i=1,2,3 .
$$

These satisfy the usual commutation relations of the form

$$
\left[\mathbf{L}_{i}, \mathbf{x}_{j}\right]=i \hbar \epsilon_{i j k} \mathbf{x}_{k}, \quad\left[\mathbf{L}_{i}, \mathbf{p}_{j}\right]=i \hbar \epsilon_{i j k} \mathbf{p}_{k} .
$$

The GUP can be established in this context if we take into account the presence of the minimal length scale. Considering physical states with $\langle\mathbf{p}\rangle=0$ and the fact that the momentum uncertainties $\Delta \mathbf{p}_{i}$ are isotropic, we see from
(1) and (2) that the Heisenberg uncertainty principle takes a modified form (GUP) that is given by

$$
\Delta \mathbf{x}_{i} \Delta \mathbf{p}_{i} \geq \frac{\hbar}{2}\left(1+3 \beta\left(\Delta \mathbf{p}_{i}\right)^{2}+\beta^{\prime}\left(\Delta \mathbf{p}_{i}\right)^{2}\right) .
$$

Hence, the specific correction of the quantum commutation relations between $\mathbf{x}_{i}$ and $\mathbf{p}_{i}$ leads to the extension of the usual uncertainty relations clarifying a lower bound. The additional terms in this principle are the consequence of the modification of ordinary space of position and momentum, which is nonnegligible especially in the high energy quantum regime with the energy comparable to the Planck mass $\sqrt{\hbar c / G} \sim 10^{19} \mathrm{GeV}$. Such GUP is also obtained by analyzing the Gedanken experiment $[43,44]$.

By minimizing position uncertainty with respect to $\Delta \mathbf{p}_{i}$ in the limit that GUP is saturated, we obtain an isotropic minimal length such that

$$
\Delta \mathbf{x}_{\min }=\hbar \sqrt{3 \beta+\beta^{\prime}} .
$$

This relation implies that there is a loss of the notion of localization in the position space. Since we are going to work in momentum space, it is convenient to use the following representation of the position and momentum operators:

$$
\begin{gathered}
\mathbf{x}_{i}=i \hbar\left[\left(1+\beta p^{2}\right) \frac{\partial}{\partial p_{i}}+\beta^{\prime} p_{i} p_{j} \frac{\partial}{\partial p_{j}}+\gamma p_{i}\right], \\
\mathbf{p}_{i}=p_{i} \\
\mathbf{L}_{i}=-i \hbar \epsilon_{i j k} p_{j} \frac{\partial}{\partial p_{k}} .
\end{gathered}
$$

The parameter $\gamma$ can be taken arbitrarily, and it just modifies the squeezing factor of the momentum space measure without affecting the commutation relations. In fact, the inner product is now defined as

$$
\int \frac{d^{3} p}{\left[1+\left(\beta+\beta^{\prime}\right) p^{2}\right]^{1-\left(\left(\gamma-\beta^{\prime}\right) /\left(\beta+\beta^{\prime}\right)\right)}}|p\rangle\langle p|=1 .
$$

\section{The Dirac Oscillator with Minimal Length}

The modification of the wave function and the quantum energy that has taken place due to the existence of minimal length will be investigated here by a rigorous procedure for their evaluation. To do this, we consider momentum space problem which is more easier than that in position space due to the fact that position operators do not commute each other [see, (2)]. The replacement $\mathbf{p} \rightarrow \mathbf{p}-i \widetilde{\beta} m \omega \mathbf{r}$ in the Dirac equation for a free particle gives the Dirac oscillator equation [42] such that

$$
\left[c \widetilde{\alpha}(\mathbf{p}-i \widetilde{\beta} m \omega \mathbf{r})+\widetilde{\beta} m c^{2}\right] \psi=W \psi,
$$

where $m$ is the rest mass, $\omega$ is the frequency of the Dirac oscillator, $\widetilde{\alpha}$ and $\widetilde{\beta}$ are the Dirac matrices, and $\psi=\left(\begin{array}{l}\psi_{a} \\ \psi_{b}\end{array}\right)$ is a two-component spinor. 
Using the following representation of $\widetilde{\alpha}$ and $\widetilde{\beta}$ :

$$
\tilde{\alpha}=\left(\begin{array}{cc}
0 & \sigma \\
\sigma & 0
\end{array}\right), \quad \tilde{\beta}=\left(\begin{array}{cc}
1 & 0 \\
0 & -1
\end{array}\right),
$$

we get the following two simultaneous equations:

$$
\begin{aligned}
& W \psi_{a}(\mathbf{p})=c \sigma(\mathbf{p}+i m \omega \mathbf{r}) \psi_{b}(\mathbf{p})+m c^{2} \psi_{a}(\mathbf{p}), \\
& W \psi_{b}(\mathbf{p})=c \sigma(\mathbf{p}-i m \omega \mathbf{r}) \psi_{a}(\mathbf{p})-m c^{2} \psi_{b}(\mathbf{p}) .
\end{aligned}
$$

This system gives the following factorized equation for the large component $\psi_{a}(\mathbf{p})$ :

$$
\begin{aligned}
& \left(W^{2}-m^{2} c^{4}\right) \psi_{a}(\mathbf{p}) \\
& =c^{2}\left\{\mathbf{p}^{2}+m^{2} \omega^{2} \mathbf{r}^{2}+i m \omega[\sigma \mathbf{r}, \sigma \mathbf{p}]+i m^{2} \omega^{2} \sigma(\mathbf{r} \wedge \mathbf{r})\right\} \psi_{a}(\mathbf{p}) .
\end{aligned}
$$

Using (1) and (2), we easily show that

$$
\begin{gathered}
{[\sigma \mathbf{r}, \sigma \mathbf{p}]=i \hbar\left(1+\left(\beta+\beta^{\prime}\right) p^{2}\right)\left(\frac{2 \sigma \mathbf{L}}{\hbar}+3\right),} \\
\mathbf{r} \wedge \mathbf{r}=-i \hbar\left(2 \beta-\beta^{\prime}+\left(2 \beta+\beta^{\prime}\right) \beta p^{2}\right) \mathbf{L} .
\end{gathered}
$$

The substitution of the above equations into (13) gives

$$
\begin{gathered}
\frac{\left(W^{2}-m^{2} c^{4}\right)}{c^{2}} \psi_{a}(\mathbf{p}) \\
=\left\{\mathbf{p}^{2}+m^{2} \omega^{2} \mathbf{r}^{2}\right. \\
+\left[-2 m \omega\left(1+\left(\beta+\beta^{\prime}\right) p^{2}\right)\right. \\
\left.+m^{2} \omega^{2} \hbar\left(2 \beta-\beta^{\prime}+\left(2 \beta+\beta^{\prime}\right) \beta p^{2}\right)\right] \sigma \mathbf{L} \\
\left.-3 m \omega \hbar\left(1+\left(\beta+\beta^{\prime}\right) p^{2}\right)\right\} \psi_{a}(\mathbf{p}) .
\end{gathered}
$$

The first two terms in the right-hand side constitute the Hamiltonian of the 3-dimensional harmonic oscillator. The third term is the spin-orbit contribution with a momentum dependent coupling strength. The fourth term is a momentum-dependent shift function which affects both the energy levels and wave functions. It is obvious that the usual Dirac oscillator equation is reproduced in the limit $\beta=\beta^{\prime}=$ 0 .

3.1. Wave Functions. Let us now derive wave functions of the Dirac oscillator in momentum space by solving (15). We make the decomposition of the wave function into radial part and a spin-angular part as

$$
\psi(\mathbf{p})=\left(\begin{array}{l}
\psi_{a}(\mathbf{p}) \\
\psi_{b}(\mathbf{p})
\end{array}\right)=\left(\begin{array}{l}
F(p) \varkappa_{\kappa}^{m_{j}}(\widehat{\mathbf{p}}) \\
G(p) \varkappa_{-\kappa}^{m_{j}}(\widehat{\mathbf{p}})
\end{array}\right),
$$

where $\widehat{\mathbf{p}}=\mathbf{p} /|\mathbf{p}|$ is a unit vector. For further evaluation, we need the square of the position operator:

$$
\begin{aligned}
\mathbf{r}^{2}=-\hbar^{2}\{[ & \left.\left(1+\left(\beta+\beta^{\prime}\right) p^{2}\right) \frac{\partial}{\partial p}\right]^{2} \\
& +\left[\frac{2}{p}+2(\beta+\gamma) p\right] \times\left[\left(1+\left(\beta+\beta^{\prime}\right) p^{2}\right) \frac{\partial}{\partial \mathbf{p}}\right] \\
& -\frac{L^{2}}{p^{2}}-\left(2 \beta L^{2}-3 \gamma\right) \\
& \left.+\left[\gamma\left(3 \beta+\beta^{\prime}+\gamma\right)-\beta^{2} L^{2}\right] p^{2}\right\} .
\end{aligned}
$$

The action of $(\sigma \mathbf{L})$ on the spin-angular function $\varkappa_{\kappa}^{m_{j}}(\mathbf{p})$ results in $[45]$ :

$$
\sigma \mathbf{L} \varkappa_{\kappa}^{m_{j}}(\widehat{\mathbf{p}})=\hbar \kappa \varkappa_{\kappa}^{m_{j}}(\widehat{\mathbf{p}}),
$$

where the quantum number $\kappa$ is equal to $[s(2 j+1)-1]$.

By substituting (17) and (18) into (15), we have

$$
\begin{aligned}
& \frac{1}{c^{2}}\left(W^{2}-m^{2} c^{4}+2 m \omega \hbar \kappa c^{2}\right. \\
& \left.+3 m \omega \hbar c^{2}-m^{2} \omega^{2} \hbar^{2} \kappa c^{2}\left(2 \beta-\beta^{\prime}\right)\right) F(p) \\
& =-m^{2} \omega^{2} \hbar^{2} \\
& \times\left\{\left[\left(1+\left(\beta+\beta^{\prime}\right) p^{2}\right) \frac{\partial}{\partial p}\right]^{2}+\left[\frac{2}{p}+2(\beta+\gamma) p\right]\right. \\
& \times\left[\left(1+\left(\beta+\beta^{\prime}\right) p^{2}\right) \frac{\partial}{\partial p}\right]-\frac{L^{2}}{p^{2}}-\left(2 \beta L^{2}-3 \gamma\right) \\
& +\left[\frac{-1}{m^{2} \omega^{2} \hbar^{2}}+\gamma\left(3 \beta+\beta^{\prime}+\gamma\right)-\beta^{2} L^{2}\right. \\
& +\frac{1}{m \omega \hbar}(2 \kappa+3)\left(\beta+\beta^{\prime}\right) \\
& \left.\left.\quad-\left(2 \beta+\beta^{\prime}\right) \beta \kappa\right] p^{2}\right\} F(p) .
\end{aligned}
$$

Although this is a very complicated equation at a glance, we can simplify it via the following standard procedure. Let us first introduce a useful parameter $\xi$ in the form

$$
\xi=\frac{\rho}{\sqrt{m \omega \hbar}}
$$

where

$$
\rho=\frac{1}{\sqrt{\beta+\beta^{\prime}}} \arctan p \sqrt{\beta+\beta^{\prime}} .
$$

Then, it is able to change the variable from $p \in(0, \infty)$ to $\xi \in(0, \pi /(2 \sqrt{k}))$ where

$$
k=\sqrt{m \omega \hbar\left(\beta+\beta^{\prime}\right)} .
$$


Notice that the relation between $\xi$ and $k$ is as follows:

$$
\xi=\frac{1}{k} \arctan \left(p \sqrt{\beta+\beta^{\prime}}\right) .
$$

Now, (19) can be rewritten as

$$
\begin{aligned}
& -\frac{\zeta}{k^{2}} F(p) \\
& =\left\{\frac{1}{k^{2}} \frac{\partial^{2}}{\partial \xi^{2}}+\left[\frac{2 m \omega \hbar \sqrt{\beta+\beta^{\prime}}}{k^{2} \tan \left(\rho \sqrt{\beta+\beta^{\prime}}\right)}+\frac{2 m \omega \hbar}{k^{2} \sqrt{\beta+\beta^{\prime}}}\right.\right. \\
& \left.\quad \times \tan \left(\rho \sqrt{\beta+\beta^{\prime}}\right)\right] \frac{\partial}{\partial \rho}-\frac{m \omega \hbar}{k^{2}}\left(\beta+\beta^{\prime}\right) L^{2} \\
& \times\left[\cot \left(\rho \sqrt{\beta+\beta^{\prime}}\right)\right]^{2} \\
& -\frac{m \omega \hbar}{k^{2}}\left(2 \beta L^{2}-3 \gamma\right)+\frac{m \omega \hbar}{k^{2}\left(\beta+\beta^{\prime}\right)} \\
& \times\left[\frac{-1}{m^{2} \omega^{2} \hbar^{2}}+\gamma\left(3 \beta+\beta^{\prime}+\gamma\right)-\beta^{2} L^{2}\right. \\
& \left.\quad+\frac{1}{m \omega \hbar}(2 \kappa+3)\left(\beta+\beta^{\prime}\right)-\left(2 \beta+\beta^{\prime}\right) \beta \kappa\right] \\
& \left.\times\left[\tan \left(\rho \sqrt{\beta+\beta^{\prime}}\right)\right]^{2}\right\} F(p),
\end{aligned}
$$

where

$$
\zeta=\frac{W^{2}-m^{2} c^{4}}{m \omega \hbar c^{2}}+2 \kappa+3-m \omega \hbar \kappa\left(2 \beta-\beta^{\prime}\right) .
$$

For further simplifications, we put

$$
F=C^{\lambda+\delta} f(S)
$$

where $S$ and $C$ are given by

$$
S=\sin (k \xi), \quad C=\cos (k \xi),
$$

and $\lambda$ is a constant that will be determined later, while $\delta=$ $\gamma /\left(\beta+\beta^{\prime}\right)$. From substitution of (26) into (24), we get

$$
\begin{aligned}
& \left(1-S^{2}\right) f^{\prime \prime}-\left[(2 \lambda+1+2(1-\eta)) S-\frac{2}{S}\right] f^{\prime} \\
& +\left\{\left(\frac{\zeta}{k^{2}}-(2 \eta-1) \times L^{2}-3 \lambda\right)\right. \\
& -\frac{L^{2}}{S^{2}}+\left(\lambda^{2}-\lambda(1+2 \eta)-\eta^{2} L^{2}+\frac{(2 \kappa+3)}{k^{2}}\right. \\
& \left.\left.-\eta(\eta+1) \kappa-\frac{1}{k^{4}}\right) \frac{S^{2}}{C^{2}}\right\} f=0,
\end{aligned}
$$

where $\eta=\beta /\left(\beta+\beta^{\prime}\right)$. At this stage, we eliminate the centrifugal barrier by imposing a condition that $\lambda$ satisfies equation

$$
\lambda^{2}-\lambda(1+2 \eta)-\eta^{2} L^{2}+\frac{(2 \kappa+3)}{k^{2}}-\eta(\eta+1) \kappa-\frac{1}{k^{4}}=0
$$

The corresponding solutions are

$$
\begin{aligned}
\lambda_{ \pm}= & \frac{1+2 \eta}{2} \\
& \pm \sqrt{\frac{(1+2 \eta)^{2}}{4}+\eta^{2} L^{2}-\frac{(2 \kappa+3)}{k^{2}}+\eta(\eta+1) \kappa+\frac{1}{k^{4}}} .
\end{aligned}
$$

To proceed further, it is convenient to introduce a parameter as $z=2 S^{2}-1$ and to choose $f(S)=S^{l} g(S)$. Then (28) becomes

$$
\begin{aligned}
& \left(1-z^{2}\right) g^{\prime \prime}(z)+[(b-a)-(a+b+2) z] g^{\prime}(z) \\
& \quad+\frac{1}{4}\left[\frac{\zeta}{k^{2}}-2 \eta L^{2}-(2 l+3) \lambda+l(2 \eta-1)\right] g(z)=0
\end{aligned}
$$

where the parameters $a$ and $b$ are defined by

$$
a=\lambda_{+}-\eta-\frac{1}{2}, \quad b=\frac{1}{2}+l
$$

Notice that $a$ is written in terms of $\lambda_{+}$given in (30), and it will be justified later. To solve this differential equation, we set

$$
\begin{gathered}
\frac{1}{4}\left[\frac{\zeta}{k^{2}}-2 \eta L^{2}-(2 l+3) \lambda+l(2 \eta-1)\right] \\
=n^{\prime}\left(n^{\prime}+a+b+1\right),
\end{gathered}
$$

where $n^{\prime}=(n-l) / 2$. This is the spectral condition from which we can extract the energy spectrum. Thus, we obtain

$$
\begin{gathered}
\left(1-z^{2}\right) g^{\prime \prime}(z)+[(b-a)-(a+b+2) z] g^{\prime}(z) \\
+n^{\prime}\left(n^{\prime}+a+b+1\right) g(z)=0,
\end{gathered}
$$

whose corresponding solution is given in terms of the Jacobi polynomials:

$$
g(z)=N P_{n^{\prime}}^{(a, b)}(z)
$$

with a normalization constant $N$.

Then, the large radial component momentum wave function is given by

$$
\begin{aligned}
& F(z) \\
& =N 2^{-(a+b+\eta+\delta) / 2}(1-z)^{(a+\eta+\delta+1 / 2) / 2}(1+z)^{(b-1 / 2) / 2} P_{n^{\prime}}^{(a, b)}(z) .
\end{aligned}
$$


Upon returning to the old variable $p$, we have

$$
\begin{aligned}
F(p)= & N\left[1+\left(\beta+\beta^{\prime}\right) p^{2}\right]^{-(a+b+\eta+\delta) / 2}\left(\sqrt{\beta+\beta^{\prime}} p\right)^{b-(1 / 2)} \\
& \times P_{n^{\prime}}^{(a, b)}\left(\frac{\left(\beta+\beta^{\prime}\right) p^{2}-1}{\left(\beta+\beta^{\prime}\right) p^{2}+1}\right),
\end{aligned}
$$

which leads to the large component of the DO wave function:

$$
\begin{aligned}
\psi_{a}(\mathbf{p})= & N\left[1+\left(\beta+\beta^{\prime}\right) p^{2}\right]^{-(a+b+\eta+\delta) / 2}\left(\sqrt{\beta+\beta^{\prime}} p\right)^{b-(1 / 2)} \\
& \times P_{n^{\prime}}^{(a, b)}\left(\frac{\left(\beta+\beta^{\prime}\right) p^{2}-1}{\left(\beta+\beta^{\prime}\right) p^{2}+1}\right) \varkappa_{\mathcal{K}}^{m_{j}}(\widehat{\mathbf{p}}) .
\end{aligned}
$$

Let us now turn to the calculation of the small component of the DO wave function $\psi_{b}(\mathbf{p})$ given by

$$
\psi_{b}(\mathbf{p})=\frac{c \sigma(\mathbf{p}-i m \omega \mathbf{r})}{W+m c^{2}} \psi_{a}(\mathbf{p}) .
$$

With the aid of the following relations [14]:

$$
\begin{gathered}
\sigma \mathbf{r}=i \hbar \sigma_{i}\left(\left(1+\beta p^{2}\right) \frac{\partial}{\partial p_{i}}+\beta^{\prime} p_{i} p_{j} \frac{\partial}{\partial p_{j}}+\gamma p_{i}\right), \\
\sigma_{i} \frac{\partial}{\partial p_{i}}=\left(\frac{\partial}{\partial p}+\frac{\sigma \mathbf{L}+2}{p}\right) \sigma_{p}=\sigma_{p}\left(\frac{\partial}{\partial p}-\frac{\sigma \mathbf{L}}{p}\right), \\
\sigma_{i} p_{i} p_{j} \frac{\partial}{\partial p_{j}}=(\sigma \cdot \mathbf{p})\left(p \frac{\partial}{\partial p}\right)=\sigma_{p} p^{2} \frac{\partial}{\partial p}=p^{2} \frac{\partial}{\partial p} \sigma_{p}, \\
\frac{\partial}{\partial p} \sigma_{p}=\sigma_{p} \frac{\partial}{\partial p},
\end{gathered}
$$

where $\sigma_{p}=(\sigma \cdot \mathbf{p}) / p$, and using (18), one finds

$$
\begin{aligned}
\psi_{b}(\mathbf{p})= & \frac{c}{W+m c^{2}} \sigma_{p} \\
& \times\left[p+m \omega \hbar\left(\left(1+\left(\beta+\beta^{\prime}\right) p^{2}\right) \frac{\partial}{\partial p}\right.\right. \\
& \left.\left.-\frac{\hbar \kappa}{p}+(\gamma-\beta \hbar \kappa) p\right)\right] \psi_{a}(\mathbf{p}) .
\end{aligned}
$$

Through the introduction of the following property:

$$
\sigma_{p} \varkappa_{\kappa}^{m_{j}}(\widehat{\mathbf{p}})=-\varkappa_{-\kappa}^{m_{j}}(\widehat{\mathbf{p}})
$$

the small radial wave function $G(p)$ is represented as follows:

$$
\begin{aligned}
G(p)= & \frac{-m \omega \hbar c}{W+m c^{2}} \\
& \quad \times\left[\left(\frac{1}{m \omega \hbar}+\gamma-\beta \hbar \kappa\right) p\right. \\
& \left.\quad+\left(1+\left(\beta+\beta^{\prime}\right) p^{2}\right) \frac{\partial}{\partial p}-\frac{\hbar \kappa}{p}\right] F(p) .
\end{aligned}
$$

At this stage we use the following decomposition, which will be useful in the calculation of both $G(p)$ and the normalization constant:

$$
\begin{aligned}
& F(p)=\frac{1}{p} f^{-(\delta+\eta-1) / 2} R_{1}(p), \\
& G(p)=\frac{1}{p} f^{-(\delta+\eta-1) / 2} R_{2}(p),
\end{aligned}
$$

where $f(p)=1+\left(\beta+\beta^{\prime}\right) p^{2}$. By comparing (44) with (37), one immediately gets

$$
R_{1}(p)=N \sqrt{\beta+\beta^{\prime}} p^{b-1 / 2} p^{b+1 / 2} f^{-(a+b+1) / 2} P_{n^{\prime}}^{(a, b)}(z)
$$

In the meantime, $R_{2}(p)$ is different depending on spin. Using the properties of the Jacobi polynomials given in [46], we have the following results for each spin:

$$
\text { (i) } s=1 / 2
$$

$$
\begin{aligned}
R_{2}(p)= & \frac{-m \omega \hbar c N \sqrt{\beta+\beta^{\prime}}}{W+1 / 2} \\
& \times\left[f \frac{\partial}{\partial p}+\left(\frac{1}{m \omega \hbar}-\beta \hbar \kappa\right) p-\frac{\hbar \kappa}{p}\right] \\
& \times p^{b+1 / 2} f^{-(a+b+1) / 2} P_{n^{\prime}}^{(a, b)}(z) \\
= & \frac{-2 m \omega \hbar c N \sqrt{\beta+\beta^{\prime}} b-1 / 2}{W+m c^{2}} \\
& \times p^{b-1 / 2} f^{-(a+b+1) / 2}(1+z) \frac{d}{d z} P_{n^{\prime}}^{(a, b)}(z) \\
= & \frac{-2 m \omega \hbar c\left(\beta+\beta^{\prime}\right)\left(a+b+n^{\prime}+1\right) N \sqrt{\beta+\beta^{\prime}}}{W-1 / 2} \\
& \times p^{b+3 / 2} f^{-(a+b+3) / 2} P_{n^{\prime}-1}^{(a+1, b+1)}(z),
\end{aligned}
$$

(ii) $s=-1 / 2$

$$
\begin{aligned}
R_{2}(p)= & \frac{-m \omega \hbar c N \sqrt{\beta+\beta^{\prime}}}{W-1 / 2} \\
& \times\left[f \frac{\partial}{\partial p}+\left(\frac{1}{m \omega \hbar}-\beta \hbar(\kappa+1)\right) p-\frac{\hbar(\kappa+1)}{p}\right] \\
& \times p^{b+1 / 2} f^{-(a+b+1) / 2} P_{n^{\prime}}^{(a, b)}(z) \\
= & \frac{-2 m \omega \hbar c N \sqrt{\beta+\beta^{\prime}}{ }^{b-1 / 2}}{W+m c^{2}} p^{b-1 / 2} f^{-(a+b+1) / 2}
\end{aligned}
$$




$$
\begin{aligned}
& \times\left[(1+z) \frac{d}{d z}+b\right] P_{n^{\prime}}^{(a, b)}(z) \\
= & \frac{-2 m \omega \hbar c\left(b+n^{\prime}\right) N{\sqrt{\beta+\beta^{\prime}}}^{b-1 / 2}}{W+m c^{2}} \\
& \times p^{b-1 / 2} f^{-(a+b+1) / 2} P_{n^{\prime}}^{(a+1, b-1)}(z) .
\end{aligned}
$$

Now, the normalization constant $N$ can be calculated using the modified closure relation

$$
\int_{0}^{\infty} \frac{d p}{f(p)}\left(\left|R_{1}(p)\right|^{2}+\left|R_{2}(p)\right|^{2}\right)=1
$$

If we substitute (46)-(48) into (44) and (45), the final expressions of the radial components of normalized wave functions are obtained as follows:

$$
\begin{aligned}
F(p)= & \left(\frac{W+m c^{2}}{2 W}\right)^{1 / 2} \\
& \times A^{\left(n^{\prime}\right)}(a, b) p^{b-1 / 2} f^{-(1 / 2)(a+b+\eta+\delta)} P_{n^{\prime}}^{(a, b)}(z), \\
G(p)= & -\varepsilon\left(\frac{W-m c^{2}}{2 W}\right)^{1 / 2} \\
& \times A^{\left(\widetilde{n}^{\prime}\right)}(\widetilde{a}, \widetilde{b}) p^{\widetilde{b}-1 / 2} f^{-(1 / 2)(\widetilde{a}+\tilde{b}+\eta+\delta)} P_{\widetilde{n}^{\prime}}^{(\widetilde{a}, \widetilde{b})}(z),
\end{aligned}
$$

where

$$
\begin{gathered}
A^{\left(n^{\prime}\right)}(a, b) \\
=\left(\frac{2\left(\beta+\beta^{\prime}\right)^{b+1}\left(a+b+2 n^{\prime}+1\right) n^{\prime} ! \Gamma\left(a+b+n^{\prime}+1\right)}{\Gamma\left(a+n^{\prime}+1\right) \Gamma\left(b+n^{\prime}+1\right)}\right)^{1 / 2}, \\
\tilde{a}=a+1, \quad \tilde{b}=b+2 s, \quad \tilde{n}^{\prime}=n^{\prime}-s-\frac{1}{2}, \quad \epsilon=\frac{W}{|W|},
\end{gathered}
$$

and $n^{\prime}=0,1,2, \ldots$, except when $s=1 / 2$ and $\epsilon=-1$, in which case $n^{\prime}=1,2,3, \ldots$.

At this stage, we turn back to the problem of justifying that $a$ is expressed in terms of $\lambda_{+}$as shown in (32). It was pointed in [21] that the normalization condition alone does not always guarantee physically relevant wave functions for the case considered the existence of minimal length. However, the physical wave functions of our system must be recognizable in the domain of momentum since $\mathbf{p}_{i}$ and $\mathbf{p}_{j}$ commutes mutually as shown in (3). This physically means that there is a finite uncertainty in momentum, leading to the following condition:

$$
\left\langle p^{2}\right\rangle=\int_{0}^{\infty} \frac{p^{2} d p}{f(p)}\left(\left|R_{1}(p)\right|^{2}+\left|R_{2}(p)\right|^{2}\right)<\infty,
$$

or

$$
\int_{0}^{\infty} \frac{p^{2} d p}{f(p)}\left|R_{1}(p)\right|^{2}<\infty, \quad \int_{0}^{\infty} \frac{p^{2} d p}{f(p)}\left|R_{2}(p)\right|^{2}<\infty .
$$

It is easy to see that the integrand with the small radial wave function behaves like $p^{-(2 \lambda-2 \eta-1)}$ for $p \rightarrow \infty$, which requires $\lambda>\eta+(1 / 2)$. This condition can only be fulfilled by choosing the upper sign in (30). For the case $\lambda=\lambda_{+}$, the integrand with the large radial wave function behaves like $p^{-\left(2 \lambda_{+}-2 \eta+1\right)}$, and then the convergence criterion requires $\lambda_{+}>\eta-(1 / 2)$ which is automatically satisfied in our treatment.

Let us derive the nonrelativistic limit of the Dirac oscillator equation with minimal length by setting $W=m c^{2}+E$ with $E \ll m c^{2}$ and dividing by $2 m$ from (13). Then, one finds $E \psi_{a}(\mathbf{p})$

$=\left[\frac{\mathbf{p}^{2}}{2 m}+\frac{1}{2} m \omega^{2} \mathbf{r}^{2}-\mu_{S L}(p) \sigma \mathbf{L}-\frac{3}{2} \hbar \omega\left(1+\left(\beta+\beta^{\prime}\right) p^{2}\right)\right] \psi_{a}(\mathbf{p})$,

where

$$
\begin{aligned}
\mu_{S L}(p)= & \frac{\omega}{\hbar}\left(1+\left(\beta+\beta^{\prime}\right) p^{2}\right) \\
& -\frac{\omega^{2}}{4}\left(2 \beta-\beta^{\prime}+\left(2 \beta+\beta^{\prime}\right) \beta p^{2}\right) .
\end{aligned}
$$

We observe that, besides the usual shifted 3D harmonic oscillator, the Hamiltonian contains an additional interaction term that is originated from the presence of the minimal length. Unlike the usual Dirac oscillator, the second term in (54) exhibits a momentum-dependent spin-orbit coupling strength given by $\mu_{S L}(p)$. As it is well known, the spin-orbit interaction is generated by the scalar potential in the usual case. In our setup such a scalar potential can be attributed to a gravitation-like field generated by the perturbation of the space in the presence of the minimal length.

3.2. Energy Spectrum. The existence of a nonvanishing minimal length implies the modification of energy level for the usual treatment of the Dirac oscillator. In order to derive the energy spectrum of the Dirac oscillator with minimal length, we substitute the expressions of $n^{\prime}, a, b, \lambda_{+}$, and $\zeta$ into (33) and solve for $W$. Indeed, after a straightforward calculations, we obtain

$$
\begin{aligned}
& W^{2}-m^{2} c^{4} \\
& =m \omega \hbar c^{2}\left\{2\left(n+\frac{3}{2}\right)\right. \\
& \times\left[1+(m \omega \hbar)^{2}\left(\frac{\left(\beta^{\prime}+3 \beta\right)^{2}}{4}+\beta^{2} L^{2}\right)-\epsilon_{\kappa 1}\right]^{1 / 2} \\
& +m \omega \hbar\left[\left(\beta+\beta^{\prime}\right)\left(n+\frac{3}{2}\right)^{2}+\left(\beta-\beta^{\prime}\right)\right. \\
& \left.\left.\times\left(L^{2}+\frac{9}{4}\right)+\frac{3}{2} \beta^{\prime}\right]-\epsilon_{\kappa 2}\right\},
\end{aligned}
$$


where

$$
\begin{gathered}
\epsilon_{\kappa 1}=3\left(1+\frac{2}{3} \kappa\right) m \omega \hbar\left(\beta+\beta^{\prime}\right)-m^{2} \omega^{2} \hbar^{2} \beta\left(2 \beta+\beta^{\prime}\right) \kappa, \\
\epsilon_{\kappa 2}=3\left(1+\frac{2}{3} \kappa\right)-m \omega \hbar \kappa\left(2 \beta-\beta^{\prime}\right),
\end{gathered}
$$

which are the terms expressing the spin-orbit coupling. Notice that (56) does not depend on the parameter $\gamma$, it but depends on $\beta$ and $\beta^{\prime}$. By expanding (56) up to first order in $\beta$ and $\beta^{\prime}$, we get

$$
\begin{aligned}
& W^{2}=m^{2} c^{4}+m \omega \hbar c^{2} \\
& \times\left\{2\left(n-j+\frac{1}{2}\right)\left[1+\frac{m \omega \hbar \beta}{2}\left(n-j+\frac{1}{2}\right)\right]\right. \\
& +\frac{m \omega \hbar \beta^{\prime}}{2}\left(n+j+\frac{9}{2}\right) \\
& \left.+m \omega \hbar \beta^{\prime}(-4 n-2 n j-3)\right\},
\end{aligned}
$$

for $j=l+(1 / 2)$, and

$$
\begin{gathered}
W^{2}=m^{2} c^{4}+m \omega \hbar c^{2} \\
\times\left\{2\left(n+j+\frac{3}{2}\right)\left[1+\frac{m \omega \hbar \beta}{2}\left(n+j+\frac{3}{2}\right)\right]\right. \\
+\frac{m \omega \hbar \beta^{\prime}}{2}\left(n-j+\frac{7}{2}\right) \\
\left.+m \omega \hbar \beta^{\prime}(-2 n+2 n j-3)\right\},
\end{gathered}
$$

for $j=l-(1 / 2)$.

In the standard setup where $\beta, \beta^{\prime} \rightarrow 0$, we can easily show that the following equations recover the usual energy spectrum of the Dirac oscillator [45]:

$$
\begin{array}{ll}
W^{2}-m^{2} c^{4}=2 m \omega \hbar c^{2}\left[n-j+\frac{1}{2}\right], & j=l+\frac{1}{2}, \\
W^{2}-m^{2} c^{4}=2 m \omega \hbar c^{2}\left[n+j+\frac{3}{2}\right], & j=l-\frac{1}{2},
\end{array}
$$

which shows a degeneracy of all the states with $n \pm q$ and $j \pm q$ for $j=l+(1 / 2)$, where $q$ is an integer, and of all the states with $n \pm q$ and $j \mp q$ for $j=l-(1 / 2)$. As we can see from (58) and (59), a remarkable feature of the incorporation of the minimal length in the DO equation is that these degeneracy is completely removed when $\beta^{\prime} \neq 0$. From Figure 1 , we see that the intervals between adjacent energy levels that are degenerated under $\beta^{\prime}=0$ become large as $\beta^{\prime}$ grows.

It is important to note that the energy spectrum contains additional terms proportional to $n^{2}$, which indicates hard confinement. This behavior is expected since the original problem has been mapped into the motion of a point particle near the surface of a sphere which is in essence a motion in potential wells. In our setup the boundaries of the well are localized at 0 and $\pi /\left(2 \sqrt{m \omega \hbar\left(\beta+\beta^{\prime}\right)}\right)$.

The nonrelativistic limit of the energy spectrum in the presence of minimal length is obtained from (56) by setting $W=m c^{2}+W_{n r}$ with the assumption that $W_{n r} \ll m c^{2}$. From a little calculation, we get

$$
\begin{aligned}
W_{n r}=\hbar \omega\left\{\left(n+\frac{3}{2}\right)\right. & \\
\times & {\left[1+(m \omega \hbar)^{2}\left(\frac{\left(\beta^{\prime}+3 \beta\right)^{2}}{4}+\beta^{2} L^{2}\right)-\epsilon_{\kappa 1}\right]^{1 / 2} } \\
& +\frac{m \omega \hbar}{2}\left[\left(\beta+\beta^{\prime}\right)\left(n+\frac{3}{2}\right)^{2}+\left(\beta-\beta^{\prime}\right)\right. \\
& \left.\left.\times\left(L^{2}+\frac{9}{4}\right)+\frac{3}{2} \beta^{\prime}\right]-\frac{\epsilon_{\kappa 2}}{2}\right\} .
\end{aligned}
$$

If we ignore the contribution of the spin-orbit coupling $\epsilon_{\kappa 1}$ and $\epsilon_{\kappa 2}$, this exactly coincides with the 3D harmonic oscillator energy in deformed space with minimal length [24].

In order to compare our results with the ones considered up to the first order in $\beta$, that is obtained in [14], using supersymmetric quantum mechanical methods, let us expand (61) to the first order in $\beta$ and $\beta^{\prime}$ such that

$$
\begin{aligned}
W_{n r}=\hbar \omega\{ & \left(n-j+\frac{1}{2}\right) \\
& \times\left[1+\frac{m \omega \hbar \beta}{2}\left(n-j+\frac{1}{2}\right)\right] \\
& +\frac{m \omega \hbar \beta^{\prime}}{4}\left(n+j+\frac{9}{2}\right)-m \omega \hbar \beta^{\prime} \\
& \left.\times\left(2 n+n j+\frac{3}{2}\right)\right\}, \quad j=l+\frac{1}{2}, \\
W_{n r}=\hbar \omega\{ & \left(n+j+\frac{3}{2}\right) \\
& \times\left[1+\frac{m \omega \hbar \beta}{2}\left(n+j+\frac{3}{2}\right)\right] \\
& +\frac{m \omega \hbar \beta^{\prime}}{4}\left(n-j+\frac{7}{2}\right)-m \omega \hbar \beta^{\prime} \\
& \left.\times\left(n-n j+\frac{3}{2}\right)\right\}, \quad j=l-\frac{1}{2},
\end{aligned}
$$

respectively. Clearly, in the limit $\beta^{\prime}=0$, these equations become identical with the ones obtained in [14]. The discrepancy when $\beta^{\prime} \neq 0$ can be attributed to the method used by the 
authors of [14] where there is a manifest distinction between large and small values of $j$.

Now, by setting $\beta^{\prime}=0$ and $\beta=0$ from (62), one obtains the energy levels of the standard Dirac oscillator in the nonrelativistic limit as follows:

$$
\begin{array}{ll}
W_{n}=\hbar \omega\left(n-j+\frac{1}{2}\right), & j=l+\frac{1}{2}, \\
W_{n}=\hbar \omega\left(n+j+\frac{3}{2}\right), & j=l-\frac{1}{2} .
\end{array}
$$

Using $n=2 n^{\prime}+l$, we confirm that the average energy between the up-spin and the down-spin states is $\widetilde{W}_{n}=\hbar \omega\left(2 n^{\prime}+l+\right.$ $(1 / 2))$ which differs from the usual nonrelativistic harmonic oscillator eigenvalue by $\hbar \omega$, which is attributed to the spinorbit coupling. In the presence of the minimal length, the following can be shown:

$$
\begin{aligned}
\widetilde{W}_{n}=\hbar \omega\{ & \left(2 n^{\prime}+l+\frac{1}{2}\right) \\
& \times\left[1+\frac{m \omega \hbar \beta}{2}\left(2 n^{\prime}+l+\frac{1}{2}\right)\right] \\
& \left.-\frac{m \omega \hbar \beta^{\prime}}{2}\left(2 n^{\prime}+l-\frac{1}{2}\right)\right\},
\end{aligned}
$$

where additional spin-orbit-like contribution proportional to $\beta$ and $\beta^{\prime}$ appears.

\section{Conclusion}

In this paper, we have exactly solved the Dirac oscillator equation in 3 dimensions in the framework of relativistic quantum mechanics with minimal length. Quantum features of the system such as wave functions and the energy spectrum are investigated using common techniques for noncommutative geometry algebra.

Although our wave functions for $\beta \neq 0$ and $\beta^{\prime} \neq 0$ exactly coincide with the ones obtained in [14] using supersymmetric quantum mechanical (SUSYQM) formalism, it is shown that the energy spectrum we have obtained here is different from theirs. An important point of our result, that distinguishes it from previous works, is that the presence of the minimal length reveals a quadratic dependence of the energy spectrum on quantum number $n$, as well as the appearance of the usual term that is linearly dependent on $n$, implying the property of hard confinement of the system. However, for $\beta^{\prime}=0$, the energy spectrum to the first order in $\beta$ becomes the same as the one obtained in [14].

An interesting feature that we have shown in this work is that the well-known usual infinite degeneracy of the usual Dirac oscillator energy levels is completely removed by the presence of the minimal length in the case $\beta^{\prime} \neq 0$. We see from Figure 1 that all of degenerated energies under $\beta^{\prime}=0$ are splitting in the presence of $\beta^{\prime}$, and the interval between split energies becomes large as $\beta^{\prime}$ increases. It is also confirmed that, when we ignore the spin-orbit coupling, the

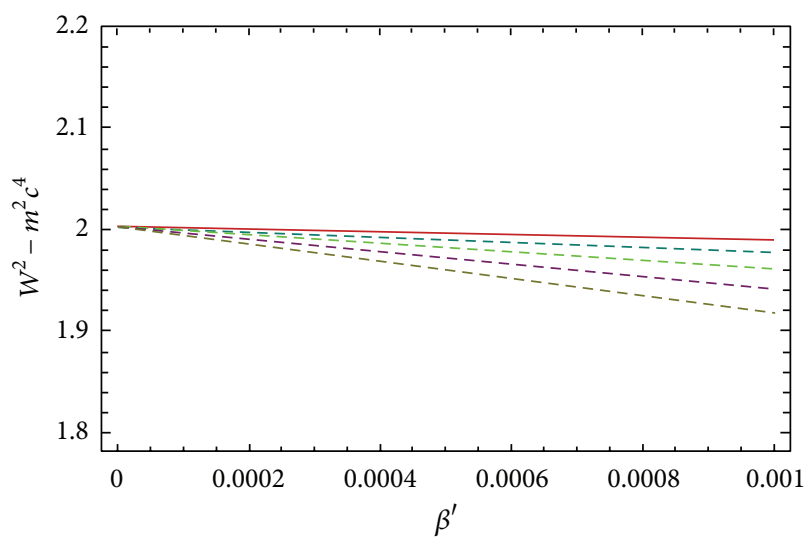

FIGURE 1: Splitting of degenerated energies in the presence of $\beta^{\prime}$. The value of $(n, l)$, in turn from upper line, are $(2,1),(3,2),(4,3),(5,4)$, and $(6,5)$. We have taken $j=l+1 / 2, \beta=0.001, c=1, m=1$, $\omega=1$, and $\hbar=1$ and all these values are chosen dimensionlessly for convenience.

nonrelativistic expression of the energy levels exactly reduces to that for the energy of 3D harmonic oscillator with the minimal length scale [24].

Finally, it is important to note that our results were obtained using a noncovariant deformed algebra given by (1)(3), and it is legitimate to ask whether the different properties of the energy spectrum obtained in this work remain valid if one uses a covariant deformed algebra like the one introduced in $[47,48]$. This issue will be addressed in our forthcoming contribution on the subject of this context. Indeed, the concept of minimal length became one of common factors for many different formulations of quantum mechanics with general relativity, due to the fact that minimal length is an intrinsic scale characterizing the physically meaningful finite minimal size in string theory.

\section{Acknowledgments}

Malika Betrouche is indebted to Professor Khireddine Nouicer (Laboratory of Theoretical Physics and the Department of Physics, University of Jijel, Algeria) for his interest his generous assistance and fruitful discussions during this work. The work of M. Maamache was supported by the Agence Thématique de Recherche en Sciences et Technologie (ATRST) contract of May 2, 2011 (code pnr: 8/u19/882). The work of J. R. Choi was supported by the Basic Science Research Program through the National Research Foundation of Korea (NRF) funded by the Ministry of Education (Grant no. 2013R1A1A2062907).

\section{References}

[1] G. Veneziano, "A stringy nature needs just two constants," Europhysics Letters, vol. 2, no. 3, pp. 199-204, 1986.

[2] D. Amati, M. Ciafaloni, and G. Veneziano, "Can spacetime be probed below the string size?” Physics Letters B, vol. 216, no. 1-2, pp. 41-47, 1989. 
[3] K. Konishi, G. Paffuti, and P. Provero, "Minimum physical length and the generalized uncertainty principle in string theory," Physics Letters B, vol. 234, no. 3, pp. 276-284, 1990.

[4] M. Kato, "Particle theories with minimum observable length and open string theory," Physics Letters B, vol. 245, no. 1, pp. 4347, 1990.

[5] R. Guida, K. Konishi, and P. Provero, "On the short distance behavior of string theories," Modern Physics Letters A, vol. 6, no. 16, pp. 1487-1504, 1991.

[6] L. J. Garay, "Quantum gravity and minimal length," International Journal of Modern Physics A, vol. 10, no. 2, pp. 145-166, 1995.

[7] S. Capozziello, G. Lambiase, and G. Scarpetta, "Generalized uncertainty principle from quantum geometry," International Journal of Theoretical Physics, vol. 39, no. 1, pp. 15-22, 2000.

[8] M. R. Douglas and N. A. Nekrasov, "Noncommutative field theory," Reviews of Modern Physics, vol. 73, no. 4, pp. 977-1029, 2001.

[9] S. Minwalla, M. Van Raamsdonk, and N. Seiberg, "Noncommutative perturbative dynamics," The Journal of High Energy Physics, vol. 2, article 020, 30 pages, 2000.

[10] R. J. Szabo, "Quantum field theory on noncommutative spaces," Physics Reports, vol. 378, no. 4, pp. 207-299, 2003.

[11] F. Scardigli, "Generalized uncertainty principle in quantum gravity from micro-black hole gedanken experiment," Physics Letters B, vol. 452, no. 1-2, pp. 39-44, 1999.

[12] F. Scardigli and R. Casadio, "Generalized uncertainty principle, extra dimensions and holography," Classical and Quantum Gravity, vol. 20, no. 18, pp. 3915-3926, 2003.

[13] Y. Chargui, A. Trabelsi, and L. Chetouani, "Exact solution of the $(1+1)$-dimensional Dirac equation with vector and scalar linear potentials in the presence of a minimal length," Physics Letters A, vol. 374, no. 4, pp. 531-534, 2010.

[14] C. Quesne and V. M. Tkachuk, "Dirac oscillator with nonzero minimal uncertainty in position," Journal of Physics A, vol. 38, no. 8, pp. 1747-1766, 2005.

[15] S. Hossenfelder, "The minimal length and large extra dimensions," Modern Physics Letters A, vol. 19, no. 37, pp. 2727-2744, 2004.

[16] S. Hossenfelder, "Running coupling with minimal length," Physical Review D, vol. 70, no. 10, Article ID 105003, 11 pages, 2004.

[17] Hossenfelder, "Suppressed black hole production from minimal length," Physics Letters B, vol. 598, no. 1-2, pp. 92-98, 2004.

[18] R. R. Sastry, "Quantum mechanics of smeared particles," Journal of Physics A, vol. 33, no. 46, pp. 8305-8318, 2000.

[19] M. Maggiore, "The algebraic structure of the generalized uncertainty principle," Physics Letters B, vol. 319, no. 1-3, pp. 83-86, 1993.

[20] A. Kempf, "Uncertainty relation in quantum mechanics with quantum group symmetry," Journal of Mathematical Physics, vol. 35, no. 9, pp. 4483-4495, 1994.

[21] A. Kempf, G. Mangano, and R. B. Mann, "Hilbert space representation of the minimal length uncertainty relation," Physical Review D, vol. 52, no. 2, pp. 1108-1118, 1995.

[22] A. Kempf, "Non-pointlike particles in harmonic oscillators," Journal of Physics A, vol. 30, no. 6, pp. 2093-2102, 1997.

[23] H. Hinrichsen and A. Kempf, "Maximal localization in the presence of minimal uncertainties in positions and in momenta," Journal of Mathematical Physics, vol. 37, no. 5, pp. 2121-2137, 1996.
[24] L. N. Chang, D. Minic, N. Okamura, and T. Takeuchi, "Exact solution of the harmonic oscillator in arbitrary dimensions with minimal length uncertainty relations," Physical Review D, vol. 65, no. 12, Article ID 125027, 8 pages, 2002.

[25] T. V. Fityo, I. O. Vakarchuk, and V. M. Tkachuk, "Onedimensional Coulomb-like problem in deformed space with minimal length," Journal of Physics A, vol. 39, no. 9, pp. 21432150, 2006.

[26] F. Brau, "Minimal length uncertainty relation and the hydrogen atom," Journal of Physics A, vol. 32, no. 44, pp. 7691-7696, 1999.

[27] R. Akhoury and Y. -P. Yao, "Minimal length uncertainty relation and the hydrogen spectrum," Physics Letters B, vol. 572, no. 1-2, pp. 37-42, 2003.

[28] S. Benczik, L. N. Chang, D. Minic, and T. Takeuchi, "Hydrogenatom spectrum under a minimal-length hypothesis," Physical Review A, vol. 72, no. 1, Article ID 012104, 4 pages, 2005.

[29] Kh. Nouicer, "Casimir effect in the presence of minimal lengths," Journal of Physics A, vol. 38, no. 46, pp. 10027-10035, 2005.

[30] U. Harbach and S. Hossenfelder, "The Casimir effect in the presence of a minimal length," Physics Letters B, vol. 632, no. 2-3, pp. 379-383, 2006.

[31] Kh. Nouicer, "Effect of minimal lengths on electron magnetism," Journal of Physics A, vol. 40, no. 9, pp. 2125-2135, 2007.

[32] Kh. Nouicer, "Pauli-Hamiltonian in the presence of minimal lengths," Journal of Mathematical Physics, vol. 47, no. 12, Article ID 122102, 11 pages, 2006.

[33] L. N. Chang, D. Minic, N. Okamura, and T. Takeuchi, "Effect of the minimal length uncertainty relation on the density of states and the cosmological constant problem," Physical Review D, vol. 65, no. 12, Article ID 125028, 7 pages, 2002.

[34] S. Benczik, L. N. Chang, D. Minic, N. Okamura, S. Rayyan, and T. Takeuchi, "Short distance versus long distance physics: the classical limit of the minimal length uncertainty relation," Physical Review D, vol. 66, no. 2, Article ID 026003, 11 pages, 2002.

[35] Kh. Nouicer, "An exact solution of the one-dimensional Dirac oscillator in the presence of minimal lengths," Journal of Physics A, vol. 39, no. 18, pp. 5125-5134, 2006.

[36] M. Moshinsky and Y. F. Smirnov, The Harmonic Oscillator in Modern Physics, Harwood, Amsterdam, 1996.

[37] R. P. Martínez-y-Romero, H. N. Núñez-Yépez, and A. L. SalasBrito, "Relativistic quantum mechanics of a Dirac oscillator," European Journal of Physics, vol. 16, no. 3, pp. 135-141, 1995.

[38] J. Benítez, R. P. Martínez y Romero, H. N. Núñez Yépez, and A. L. Salas-Brito, "Solution and hidden supersymmetry of a Dirac oscillator," Physical Review Letters, vol. 64, no. 14, pp. 1643-1645, 1990.

[39] J. Benítez, R. P. Martínez y Romero, H. N. Núñez Yépez, and A. L. Salas-Brito, "Erratum: solution and hidden supersymmetry of a Dirac oscillator, Physical Review Letters, vol. 64, pp. 16431645, 1990," Physical Review Letters, vol. 65, no. 16, pp. 20852085, 1990 .

[40] R. P. Martrinez-y-Romero, M. Moreno, and A. Zentalla, "Supersymmetric properties and stability of the Dirac sea," Physical Review D, vol. 43, no. 6, pp. 2036-2040, 1991.

[41] F. Dominguez-Adame and M. A. Gonzalez, "Solvable linear potentials in the Dirac equation," Europhysics Letters, vol. 13, no. 3, pp. 193-198, 1990.

[42] D. Ito and E. Carrieri, "An example of dynamical systems with linear trajectory," Nuovo Cimento A, vol. 51, no. 4, pp. 1119-1121, 1967. 
[43] M. Maggiore, "A generalized uncertainty principle in quantum gravity," Physics Letters B, vol. 304, no. 1-2, pp. 65-69, 1993.

[44] M. Maggiore, "Quantum groups, gravity, and the generalized uncertainty principle," Physical Review D, vol. 49, no. 10, pp. 5182-5187, 1994.

[45] P. Strange, Relativistic Quantum Mechanics, Cambridge University Press, Cambridge, UK, 1998.

[46] A. Erdélyi, W. Magnus, F. Oberhettinger, and F. G. Triconi, Higher Tran-Scendental Functions, vol. 2, McGraw-Hill, NY, USA, 1953.

[47] C. Quesne and V. M. Tkachuk, "Lorentz-covariant deformed algebra with minimal length and application to the $(1+1)$ dimensional Dirac oscillator," Journal of Physics A, vol. 39, no. 34, pp. 10909-10922, 2006.

[48] C. Quesne and V. M. Tkachuk, "Lorentz-covariant deformed algebra with minimal length," Czechoslovak Journal of Physics, vol. 56, no. 10-11, pp. 1269-1274, 2006. 

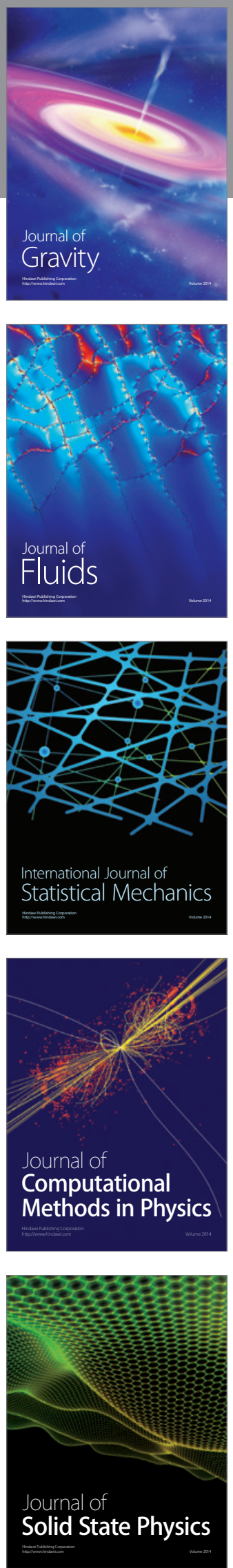

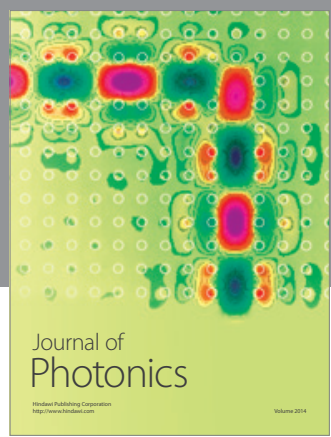

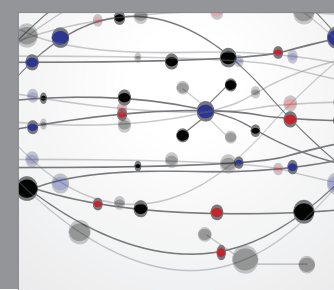

The Scientific World Journal

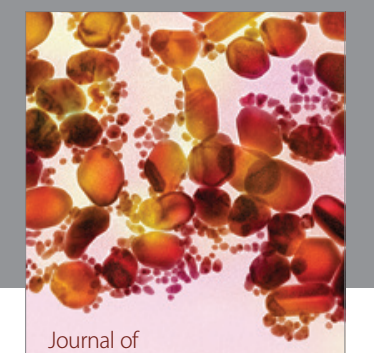

Soft Matter
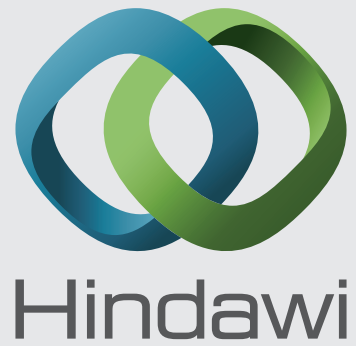

Submit your manuscripts at

http://www.hindawi.com
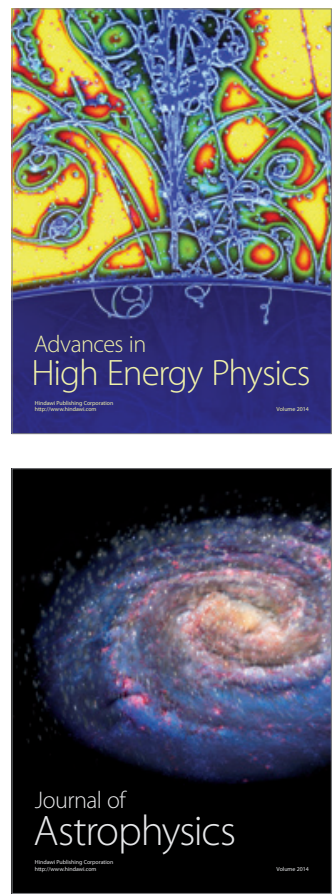
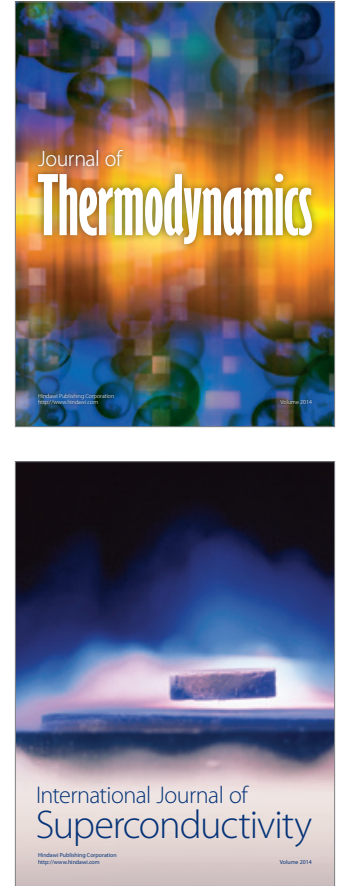
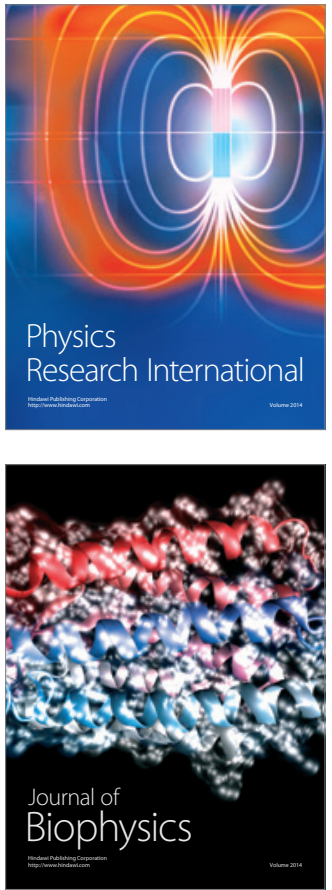
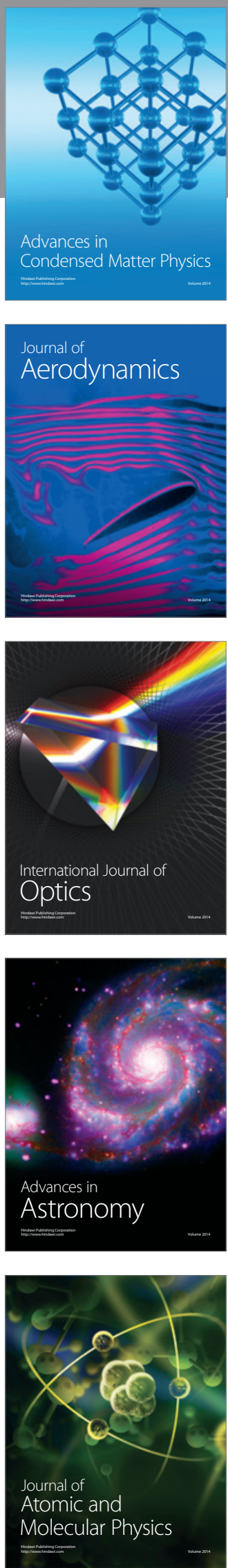